PROCEEDINGS OF THE AMERICAN MATHEMATICAL SOCIETY

Volume 127, Number 9, Pages 2805-2806

S 0002-9939(99)05370-8

Article electronically published on May 20, 1999

\title{
A SEPARABLE SPACE WITH NO SCHAUDER DECOMPOSITION
}

\author{
G. AllexANDrov, DENKA KUTZAROVA, AND A. PliCHKO
}

(Communicated by Dale Alspach)

\begin{abstract}
We combine some known results to remark that there exists a separable Banach space which fails to have a Schauder decomposition. It can be chosen as a subspace of Gowers-Maurey space without any unconditional basic sequence.
\end{abstract}

The following problem was raised in [Si] (Problem 15.1, p. 494): Does every separable Banach space have a Schauder decomposition? This question goes back to J. R. Retherford [R].

Recall that a sequence $\left\{X_{n}\right\}_{n=1}^{\infty}$ of closed subspaces of a Banach space $X$ is said to be a Schauder decomposition of $X$ if every $x \in X$ has a unique representation of the form $x=\sum_{n=1}^{\infty} x_{n}$, with $x_{n} \in X_{n}$ for every $n$.

Let $G M$ be Gowers-Maurey space which does not contain any unconditional basic sequence [GM]. As was observed by W. B. Johnson, $G M$ has in fact a stronger property, namely it is hereditarily indecomposable (H.I.); i.e., no infinitedimensional closed subspace can be written as a direct sum $Y \oplus Z$, where $Y$ and $Z$ are infinite-dimensional closed subspaces. It is known that every block subspace of $G M$ contains uniform copies of $\ell_{1}^{n}$. This follows from the lower $f$-estimate and Krivine's theorem as in [S]. Then, by Szankowski's refinement of Enflo's criterion (see [LT2, p. 111, Remark 1]), we immediately obtain the following.

Proposition. There exists a subspace $X$ of $G M$ which does not have the compact approximation property (C.A.P.).

Remark 1. For the same purpose we can as well use other H.I. spaces constructed after the breakthrough of W. T. Gowers and B. Maurey. For example, there are subspaces without the C.A.P. of the super-reflexive H.I. spaces in $[\mathrm{F}]$ in the case when they contain uniform copies of $\ell_{p}^{n}$ for $p \neq 2$. One can also use the asymptotic $\ell_{1}$ hereditarily indecomposable spaces constructed in [AD] and [ADKM]. The existence of uniform copies of $\ell_{1}^{n}$ in these spaces follows directly from the definition and one does not need to apply Krivine's theorem. Therefore, they also have subspaces without the C.A.P.

Corollary. The space $X$ is an example of a separable Banach space with no Schauder decomposition.

Received by the editors November 9, 1998.

1991 Mathematics Subject Classification. Primary 46B15.

The authors were partially supported by the Bulgarian Ministry of Education and Science under contract MM 506/95.

(C)1999 American Mathematical Society 
Proof. Assume the contrary, i.e. $X$ has a Schauder decomposition $\left\{X_{n}\right\}_{n=1}^{\infty}$.

Case 1. $\left\{X_{n}\right\}_{n=1}^{\infty}$ is a finite-dimensional decomposition. This is impossible since the existence of an F.D.D. implies B.A.P. which in turn implies C.A.P. (see [LT1]) and this contradicts the above Proposition.

Case 2. There exists $m$ such that $X_{m}$ is infinite-dimensional. Denote $Y=\left[X_{n}\right.$ : $n \neq m]$. Then $X=X_{m} \oplus Y$ which is also impossible because $X_{m}$ and $Y$ are closed infinite-dimensional subspaces of $X, X$ is a closed subspace of $G M$, and $G M$ is H.I.

Remark 2. Clearly, the result is true hereditarily in all the above mentioned H.I. spaces, e.g. we have that every subspace of $G M$ has a further subspace which has no Schauder decomposition.

\section{REFERENCES}

[AD] S. Argyros and I. Deliyanni, Examples of asymptotic $\ell_{1}$ spaces, Trans. Amer. Math. Soc. 349 (1997), 973-995. MR 97f:46021

[ADKM] S. Argyros, I. Deliyanni, D. Kutzarova and A. Manousakis, Modified mixed Tsirelson spaces, J. Funct. Anal. 159 (1998), 43-109. CMP 99:04

[GM] W. T. Gowers and B. Maurey, The unconditional basic sequence problem, J. Amer. Math. Soc. 6 (1993), 851-874. MR 94k:46021

[F] V. Ferenczi, A uniformly convex hereditarily indecomposable Banach space, Israel J. Math. 102 (1997), 199-225. MR 98m:46013

[LT1] J. Lindenstrauss and L. Tzafriri, Classical Banach spaces I, Sequence Spaces, SpringerVerlag, 1977. MR 58:17766

[LT2] J. Lindenstrauss and L. Tzafriri, Classical Banach spaces II, Function Spaces, SpringerVerlag, 1979. MR 81c:46001

[R] J. R. Retherford, Some remarks on Schauder bases II, Rev. Rouman. Math. Pure et Appl. 13 (1968), 521-527. MR 38:4969

[S] Th. Schlumprecht, An arbitrarily distortable Banach space, Israel J. Math. 76 (1991), 81-95. MR 93h:46023

[Si] I. Singer, Bases in Banach spaces II, Springer-Verlag, 1981. MR 82k:46024

Department of Mathematics and Informatics, University of Sofia, Bulgaria

Institute of Mathematics, Bulgarian Academy of Sciences, Bulgaria

Current address: Department of Mathematics, University of South Carolina, Columbia, South Carolina 29208

Department of Mathematics, Pedagogical University, Kirovograd, Ukraine 